\title{
The enhancement of amyloid precursor protein and B-site amyloid cleavage enzyme 1 interaction: Amyloid-ß production with aging
}

\author{
LIN ZOU $^{1,2}$, RONGXI YANG ${ }^{2}$, PENGHUI ZHANG $^{1}$ and YI DAI $^{3}$ \\ ${ }^{1}$ Center for Clinical Molecular Medicine, Children's Hospital, Chongqing Medical University, Chongqing 400014; \\ ${ }^{2}$ Laboratory of Molecular Cell Biology, Institute of Biochemistry and Cell Biology, Shanghai Institutes for \\ Biological Sciences, Chinese Academy of Sciences, Shanghai 200031; ${ }^{3}$ The Affiliated Hospital, \\ North Sichuan Medical College, Sichuan 617000, P.R. China
}

Received October 23, 2009; Accepted December 18, 2009

DOI: 10.3892/ijmm_00000358

\begin{abstract}
Aging is considered a high risk factor for Alzheimer's disease (AD), which is one of the most prevalent neurodegenerative disorders in the elderly population. The major pathologic feature of $\mathrm{AD}$ is senile plaques mainly containing amyloid- $\beta(A ß)$ components. However, little direct evidence has shown aging in association with $A B$. Here we show that the protein-protein interaction of amyloid precursor protein (APP) and B-site amyloid cleavage enzyme 1 (BACE1) is enhanced by the fluorescence resonance energy transfer (FRET) assay during the aging process, and the APP-BACE1 complex accumulates in the endosome in the IMR-90 fibroblast (NHF) cellular aging models. Moreover, enhanced $\mathrm{A} B$ is observed in aged cells, rat brain homogenates and human serum. Interestingly, addition of the dominant-negative mutant of Rab5, a small G-protein Rab5 involved in the endocytic process, inhibits the aging-related APP-BACE1 interaction and $A \beta$ production, suggesting that endocytosis contributes to $\mathrm{AD}$ progression.
\end{abstract}

\section{Introduction}

Amyloid plaques are one of the major pathologic features characteristic of Alzheimer's disease (AD). These plaques are mainly composed of amyloid- $\beta$ (Aß), a 38-42 amino acid peptide derived from sequential cleavages of constitutively expressed amyloid precursor protein (APP) via $\beta$ - and $\gamma$-secretases $(1,2)$. $\beta$-site amyloid cleavage enzyme (BACE) has two homologues, with BACE1 being the main $B$-secretase in the brain and the first cleavage enzyme in the production of $A B$ $(3,4)$. Recent evidence shows that the proteolytic processing of

Correspondence to: Professor Lin Zou, Center for Clinical Molecular Medicine, Children's Hospital, Chongqing Medical University, Chongqing 400014, P.R. China

E-mail: zoulin_74@yahoo.com.cn

Key words: amyloid precursor protein, $\beta$-site amyloid cleavage enzyme 1 , amyloid- $\beta$, aging, interaction
APP by 3 - and $\gamma$-secretases is correlated with the process of aging (5).

Aging is the prevailing risk factor of AD. Currently, $A B$ is known to be produced by APP through proteolytic activities of $\beta$ - and $\gamma$-secretases and by dysregulation of APP metabolism in the elderly brain $(2,6)$. While the proteolytic cleavage of APP by BACE1 is interaction-dependent, the specific biochemical mechanism and regulation of this association is unknown (7). Therefore, the purpose of this study was to determine the state of APP-BACE1 protein interaction and how this interaction correlates with aging.

Normal human IMR-90 fibroblasts (NHFs) are a wellestablished model of cellular aging. In cell culture, these cells undergo a limited number of population doubling levels (PDLs) before entering irreversible growth arrest (8). At the end-stage of their mitotic life span in vitro, fibroblasts can be maintained and though they remain metabolically active they cannot be driven into further cell division cycles (9). With this departure from cell division, multiple age-dependent cellular alterations have been described in NHFs, morphological changes with enlarged cell size, dysregulation of protein degradation, posttranslational modification of proteins, and unresponsiveness to external stimuli $(10,11)$.

Here, we utilized NHFs to investigate the alteration of APP-BACE1 protein interaction with the process of aging and found that the APP-BACE1 protein interaction and AB secretion is elevated in aging cells. Furthermore, the enhancement of $A B$ secretion is also present in aged rat brain homogenates and human serum. Moreover, the dominant negative mutant of Rab5 is able to suppress the observed age-dependent increase of APP-BACE1 interaction and Aß production, strongly indicating age-dependent endocytic dysfunction in the pathological progression of $\mathrm{AD}$.

\section{Materials and methods}

Antibodies and plasmids. Antibodies used in this study included polyclonal anti-BACE1 from Calbiochem (San Diego, CA); monoclonal anti-BACE1 and anti-6E10 against Aß from Chemicon (Temecula, CA); anti-HA (rabbit), anti-c-myc (mouse), anti-flag (Rabbit), anti-APP-CTF, from Sigma- 
Aldrich (St. Louis, MO); anti-EEA-1 and anti-LAMP-1 from BD Biosciences (San Jose, CA). The secondary antibodies were Cy3-conjugated goat anti-rabbit, Cy3-conjugated goat anti-mouse, FITC-conjugated goat anti-mouse, FITC-conjugated donkey anti-goat and Cy5-conjugated anti-mouse $\mathrm{IgG}$ from Jackson Immunoresearch (West Grove, PA). Plasmids were 3'myc-APP, 3'HA-APPsw, 3'HA-BACE1, 5'flag-Rab5S34N, 5'flag-Rab7T22N as previously reported (12).

Cell culture and transfection. NHFs were obtained from the American Type Culture Collection (Rockville, MD), maintained in DMEM medium (Gibco-BRL, Gaithersburg, MD) supplemented with $10 \% \mathrm{FBS}$ and antibiotics at $37^{\circ} \mathrm{C}$ in $5 \% \mathrm{CO}_{2}, 1 \mathrm{mM}$ sodium pyruvate (Invitrogen, Carlsbad, CA), and $10 \%$ fetal calf serum (HyClone, Logan, UT). These deficient culture conditions accelerated aging without influencing the general aged phenotype, as described previously (13). Cells were passaged by trypsinization. The PDLs were calculated as $(\log C h-\log C$ s) $/ \log (2)$, where $C h$ and $C$ s are defined as the cell number harvested and seeded, respectively. The aged phenotype was identified by senescence-associated ß-galactosidase staining assay (Cell Signaling, Danvers, MA), following the manufacturer's instructions. NHFs were transfected with Lipofectamine 2000 (Invitrogen, Carlsbad, CA).

Human serum collection and rat brain homogenates. Human serum was collected from $>20$ year-old healthy volunteers. Four groups were collected: $21-40,41-60,61-80,>80$ year-old. The subjects had no indication of amyloidosis, nor any sign of proteinuria, and were not under any medication, including colchicines, prior to the time of study. Rats of various ages were anesthetized, their hearts perfused, and the hippocampi were harvested and homogenized, centrifuged, and the supernatant was used for detecting $A ß$. Animal experiments were performed according to the institutional ethics guidelines.

Immunofluorescence and fluorescence resonance energy transfer (FRET). NHFs grown on coverglass were transfected with the plasmids described below. After $36 \mathrm{~h}$, cells were fixed by ice cold $4 \%$ PFA, washed and incubated with primary antibodies at $4^{\circ} \mathrm{C}$ overnight, then with fluorophore-conjugated secondary antibodies, and then observed with a TCS NT laser confocal microscope (Leica Microsystems, Bensheim, Germany). The krypton-argon laser (emission 488 and $543 \mathrm{~nm}$ ) was used to excite the fluorescein of donor (FITC) and acceptor (Cy3), respectively. FRET efficiency of cells was determined by using Leica software. Selected intracellular areas of the acceptor molecules (Cy3) were photobleached. FRET efficiency was then calculated as follows: (1-[Donor (FITC)/ pre-bleach/Donor (FITC)/post-bleach]) X 100\%. Negative control cells were NHFs co-transfected with 3'myc-APP and 3'HA-APPsw, which showed complete co-localization but no FRET. Positive control cells were transfected with 3 'myc-APP, fixed and incubated with mouse anti-myc, then labeled with Cy3-conjugated mouse anti-goat, followed by FITC-conjugated donkey anti-goat. This positive control provided an 'upper limit' of FRET value for the system.

ELISA for A $\beta$ production. After incubated in $500 \mu 1$ conditioned medium overnight, cell secretion of $A ß 40$ and
Aß42 was quantified, respectively, using sandwich ELISA kits for human Aß40, Aß42 and SAPPß (BioSource International, Camarillo, CA). For rat, the sandwich ELISA kit from Wako was used (Osaka, Japan). The final values were normalized to the fold. Each experiment was repeated three times.

Statistical analysis. Data were analyzed by Student's t-test for comparison of independent means, with pooled estimates of common variances, $\mathrm{p}<0.05$ was considered significant.

\section{Results}

Age-dependent increases in APP-BACE1 protein-protein interactions. $\mathrm{A} B$ is reported to be increased in both healthy persons and AD patients, and the APP-BACE1 protein interaction is critical for $A B$ increase (14). We used NHFs as a cellular model of aging to study age-related APP-BACE1 interactions. Nearly $100 \%$ NHFs entered a state of irreversible growth arrest at PDLs 45 , which was measured by senescenceassociated B-galactosidase stained (data not shown) and consistent with a previously-described aged cell phenotype (11). By utilizing the nondestructive FRET assay to detect protein-protein interactions, we were able to specifically measure the extent of APP-BACE1 associations in an agedependent fashion. 3'HA-BACE1 and 3'myc-APP were cotransfected into cells, then labeled by $\mathrm{Cy} 3$ (red) and FITC (green), respectively, and both these constructs showed strong co-localization within the cytoplasm at PDLs 39 (Fig. 1A, left panel). We detected an increase in APP fluorescence after photobleaching areas with $543 \mathrm{~nm}$ argon laser to quench Cy3-labeled 3'HA-BACE1, thus indicating FRET efficiency (Fig. 1A, right panel). FRET signal efficiency increased in an age-dependent manner from PDLs $25(17.38 \pm 3.04 \%, \mathrm{n}=18)$ to PDLs $39(60.99 \pm 6.09 \%, n=18)$ (Fig. 1B and C), indicating that APP-BACE1 protein-protein interaction was directly enhanced as a consequence of the cellular aging process.

Increased APP-BACE1 complex localizes to the endosome in aged cells. Given that the endosome is a primary site of APP cleavage by BACE1 $(3,15)$, we sought to determine if the localization of APP-BACE1 complex also occurred in an agedependent manner. 3'HA-BACE1 and 3'myc-APP expression constructs were co-transfected into different-aged NHFs as shown in Fig. 1. Localization was determined by co-staining for anti-HA or anti-myc with various markers for cell organelles. We found co-localization of signal from EEA-1 (early endosome antigen-1), an endosomal marker (16) with myc-APP and HA-BACE1 (Fig. 2A). In addition, this co-localization increased in an age-dependent manner, with a peak co-

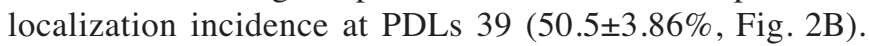
The transfection efficiency was similar in all passages of NHFs (data not shown). However, the co-localization of myc-APP, HA-BACE1 and lysosomal marker LAMP-1 (14) had no obvious change with regard to age (Fig. $2 \mathrm{C}$ and D), indicative that alteration of APP-BACE1 co-localization during the aging process is primarily in the endosome, which is the key subcellular site of $\mathrm{A} ß$ production $(3,15)$.

Age-dependent increases in cellular A $\beta$ production. Given our data regarding co-localization of APP and BACE1 occurring 
A

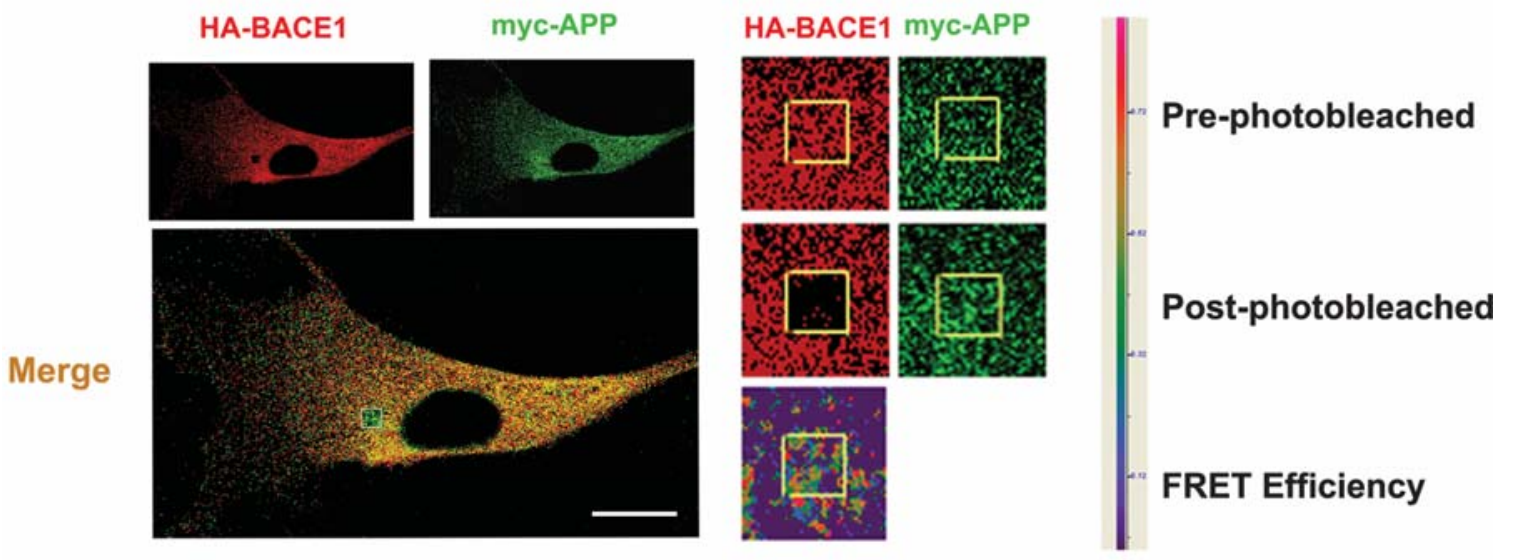

B

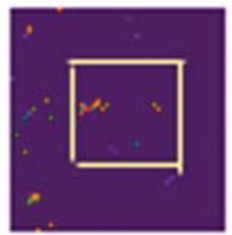

PDLs 25

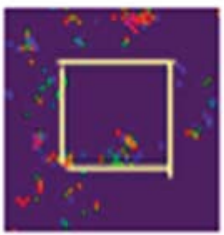

29

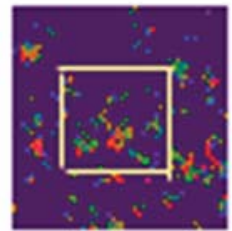

33

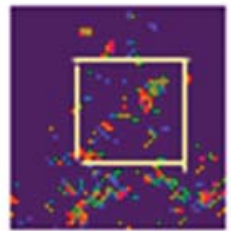

39

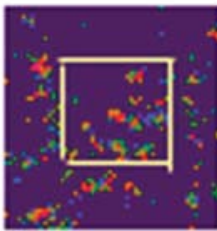

45

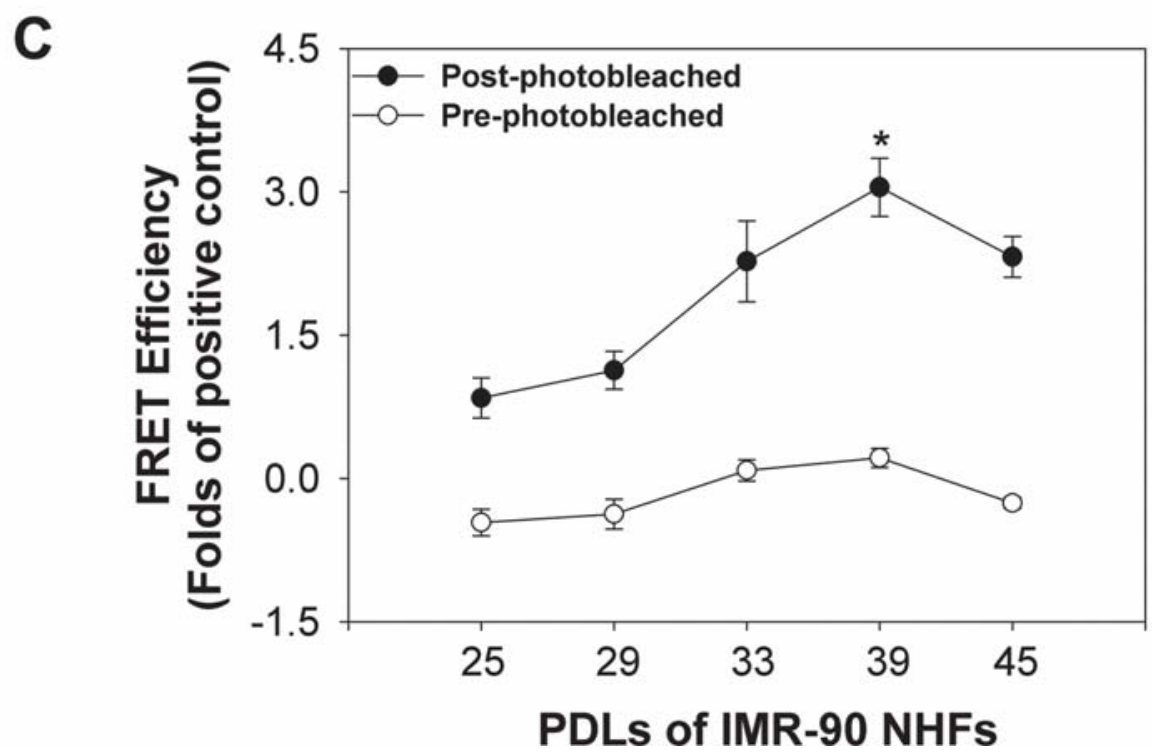

Figure 1. Age-dependent increase in APP-BACE1 protein-protein interactions. NHFs were co-transfected with 3'HA-BACE1 (immunostained by anti-HA, Cy3, red) and 3 'myc-APP (immunostained by anti-myc, FITC, green) for $36 \mathrm{~h}$. Photobleach of Cy3 labeled BACE1 led to a remarkable increase in the APP (FITC) fluorescent signal in the photobleached area, demonstrated FRET (boxed area). A. Representative figure of FRET between APP and BACE1 in the cytoplasm of NHFs at PDLs 39. Merged figure (left) show NHFs after photobleached, the region of photobleach was indicated by the white outlined box. Scale bar, $10 \mu \mathrm{m}$. Images on the right panel showed pre- and post-photobleached and FRET efficiency of the boxed area. B. Representative FRET efficiency figures of NHFs at PDLs 25, 29, 33, 39 and 45 respectively. C. Average FRET efficiency (fold of the positive control) of the bleached and unbleached regions in different-aged NHFs co-expressed 3'myc-APP and 3'HA-BACE1. PDLs 25 ( $n=18$ ); PDLs 29 ( $n=33$ ); PDLs 33 ( $n=22$ ); PDLs 39 ( $n=18$ ); and PDLs 45 (n=24). Data of positive and negative control of respective passage are not shown. ${ }^{*} \mathrm{P}<0.001$ vs. PDLs 25.

within endosomes, we next looked for age-dependent changes of $A B$ secretion rates in cells. ELISA assay was used for secreted $A B$ with co-transfected NHFs of varying age. We found that the secretion of both $A B 40$ and $A B 42$ gradually increased from NHFs at PDLs 29 and NHFs peaked at PDLs 39, with obvious Aß40 rising levels (Fig. 3A). We also observed a similar trend of increase in $\beta$-cleaved ectodomain protein ( $\mathrm{AAPP} \beta$, data not shown). In addition, using isolated 

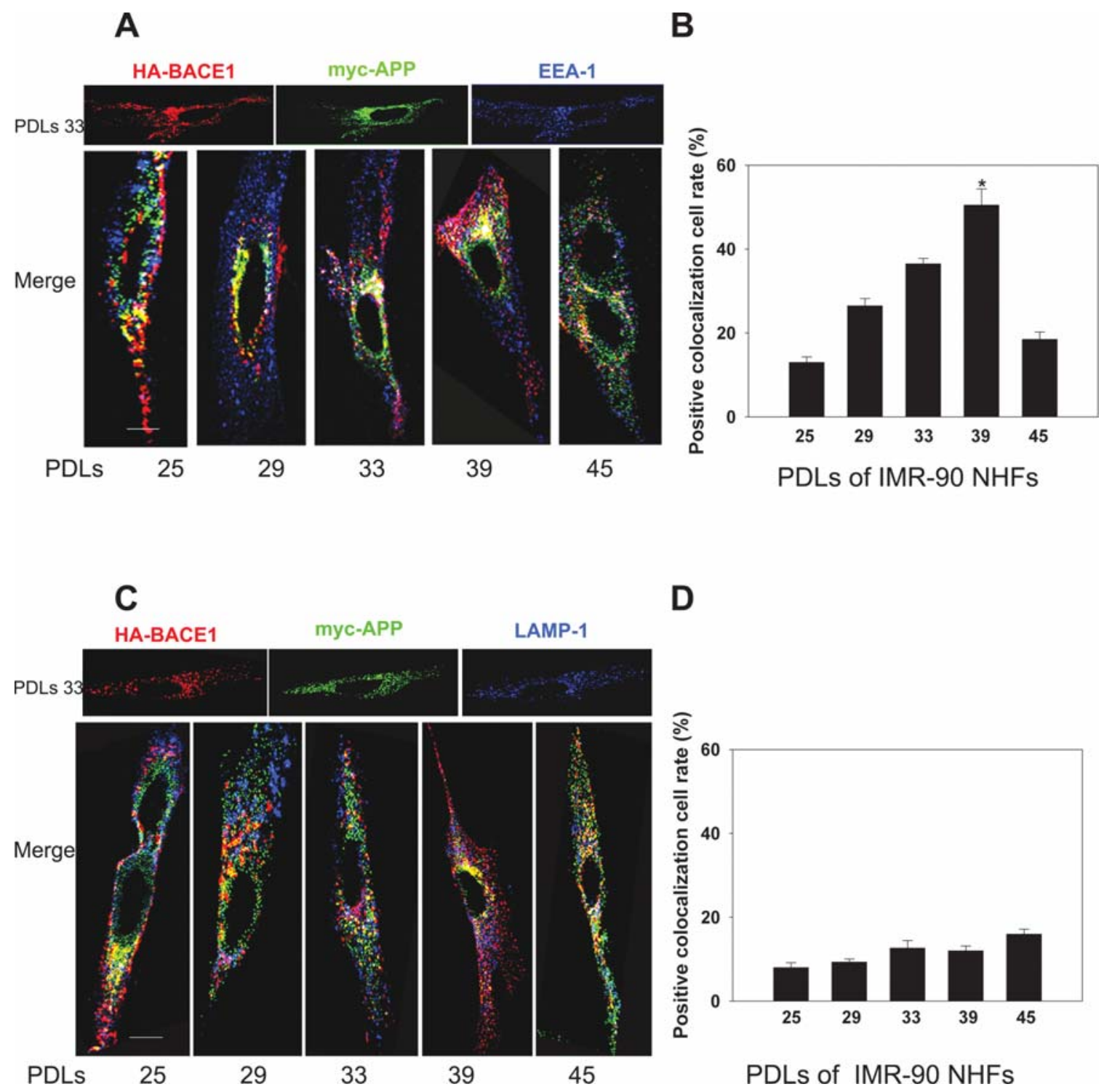

Figure 2. Increased APP-BACE1 complex localizes to the endosome in aged cells. A. NHFs of varying-age were co-transfected with 3'HA-BACE1 and 3 'myc-APP for $36 \mathrm{~h}$, then 3'HA-BACE1 and 3'myc-APP were labeled and stained by Cy3 (red) and FITC (green) respectively. EEA-1 was stained by Cy5 (blue). C. 3'HA-BACE1 and 3'myc-APP in NHFs were stained as described as in A. LAMP-1 was stained by Cy5 (blue). B. Statistical graph of the positive co-localizatoin ratio of BACE1, APP and endosome shown in A and D. BACE1, APP and lysosome shown in C in 100 transfected cells at each passage respectively. Scale bar, $40 \mu \mathrm{m}$. Data are representative of at least three independent experiments. ${ }^{*} \mathrm{P}<0.001 \mathrm{vs.}$. PDLs 25.

hippocampi from varying-aged rats, we found that both $\mathrm{A} \beta 40$ and $\mathrm{A} B 42$ levels were increased in 24 month-old rats (1.393 \pm 0.027 - and $1.305 \pm 0.095$-fold, respectively) vs. 12 month-old counterparts (Fig. 3B). Furthermore, in human serum samples, $A ß 40$ and $A ß 42$ levels, when normalized to the 41-60 year-old group, increased with age, and the highest levels were found in the $>80$ year-old group (Fig. 3C).

Role of endocytosis in APP-BACE1 age-dependent protein interactions. BACE1 activity, particularly when it pertains to cleavage of APP, appears to require endocytosis $(3,7,17)$. To confirm this, we utilized dominant negative mutant constructs of the small G-protein Rab5, which has been previously shown to hinder the endocytic process (26). 5'flag-Rab5S34N dominant-negative mutant constructs were co-transfected with both 3'myc-APP and 3'HA-BACE1 in NHFs. As a result, APP-BACE1 FRET efficiency was markedly reduced in cells transfected with Rab5S34N dominant negative mutant $(17.27 \pm 4.71 \%, \mathrm{n}=16)$ vs. $\beta$-gal co-transfected controls $(57.11 \pm 5.16 \%, \mathrm{n}=13)$, as well as compared with cells transfected with Rab7T22N $(45.39 \pm 5.60 \%, n=17)$, the dominant negative mutant for lysosome formation (Fig. 4A) (12). Furthermore, unlike controls or Rab7T22N-transfected cells, we found that Rab5S34N-transfected cells had no APPBACE1-EEA1 co-localization (Fig. 4B) or Aß secretion (Fig. 4C), thus supporting a clear role for endocytosis in these processes.

\section{Discussion}

Aging is viewed as the most significant risk factor for AD, and closely correlates with AD neuropathology. Mounting evidence shows that the majority of pathologic AD's features, including amyloid plaques, neurofibrillary tangle (NFT), 
A

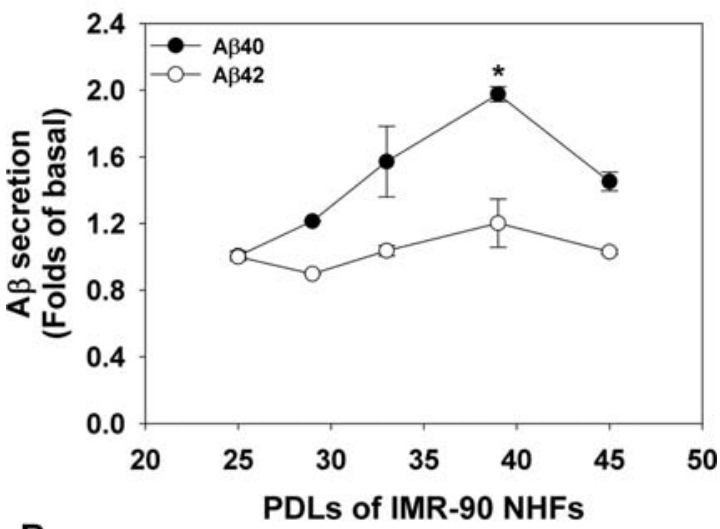

B

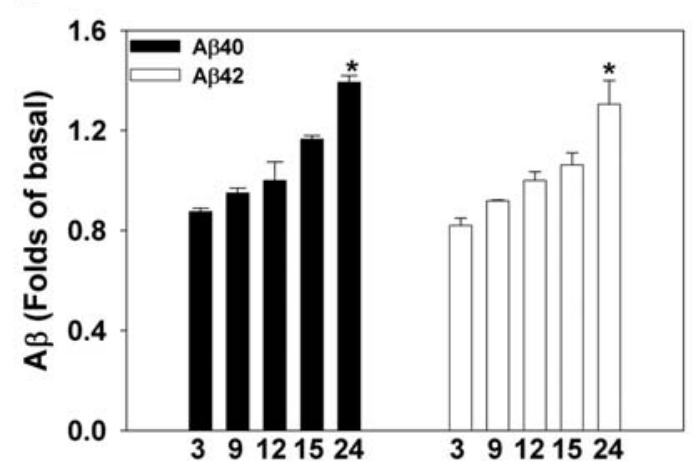

Rat Age (Months)

C

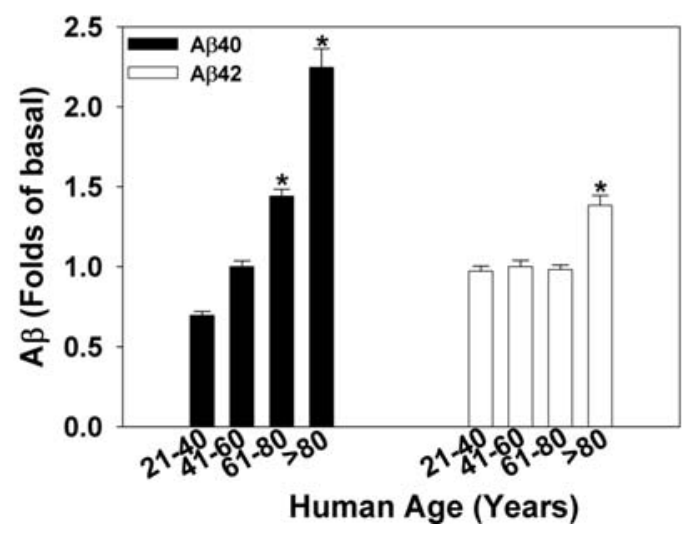

Figure 3. Age-dependent increases in Amyloid-beta production. A. NHFs of varying-age were transfected with 3'HA-APP, $48 \mathrm{~h}$ later, then accumulated cells with $500 \mu 1$ conditional media overnight, harvested the accumulated media to detect the secreted Aß40 and Aß42 level with ELISA. The number represented as fold vs. the levels in PDLs 25 (as 1.0-fold). Each was repeated three times and obtained mean \pm SE. ${ }^{*} \mathrm{P}<0.001$ vs. PDLs 25 . B. Rat hippocampi of various ages were collected, homogenized, centrifuged. Quantified protein and supernatant were used (equal amount of total protein) to detect secreted $A \beta 40$ and AB42 levels with ELISA (Wako, n=6). The number represented as the fold vs. level of 12 month-old rats (as 1.0-fold). ${ }^{*} \mathrm{P}<0.001$ vs. 9 month-old rats. C. Human serum was separated from periphery blood in persons of varying ages, and then $A B 40$ and $A ß 42$ levels were detected with ELISA. Each group included 20 persons (10 male and 10 female), repeated three times and got mean \pm SE. The number represented as fold compared with the level of 41-60 year-old group (as 1.0-fold). ${ }^{*} \mathrm{P}<0.001$ vs. $41-60$ year-old group.

neuronal death, and synaptic loss are more prevalent in the brains of elderly patients when compared to younger persons (18), highly suggestive that aging contributes to the acceleration
A
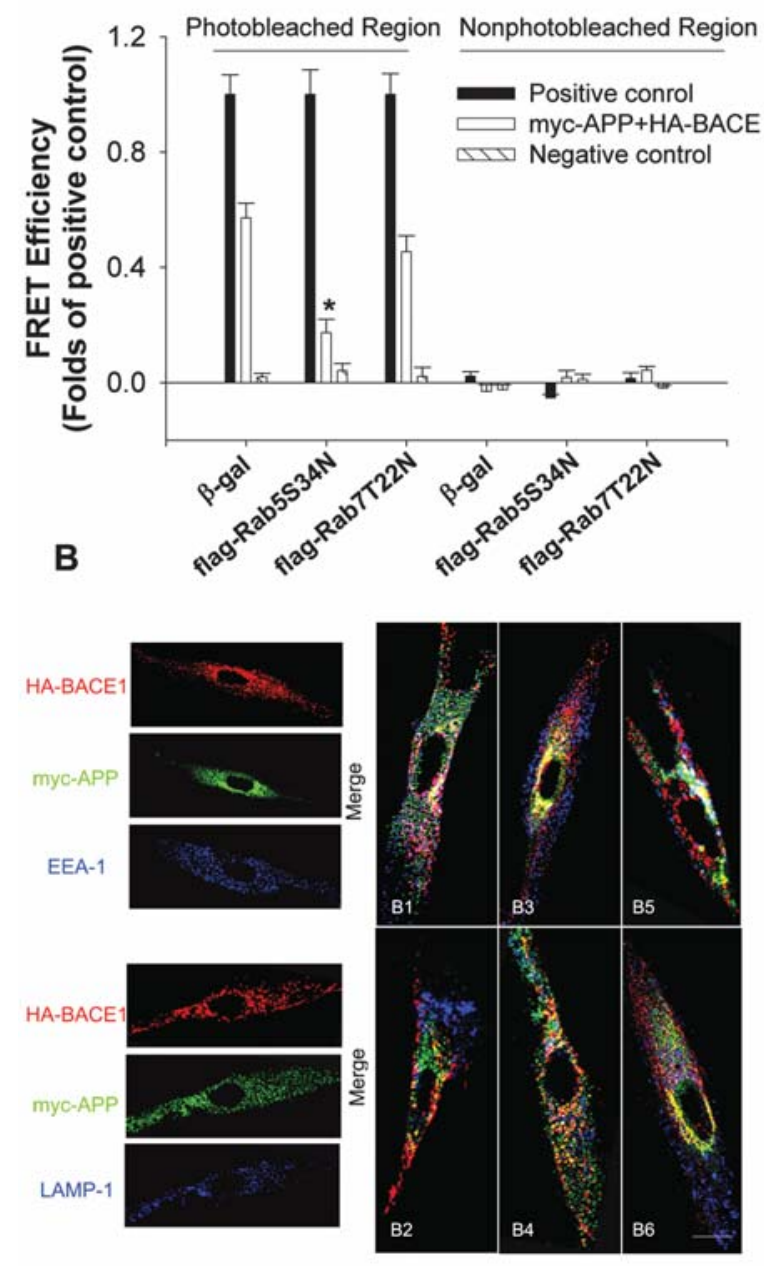

C

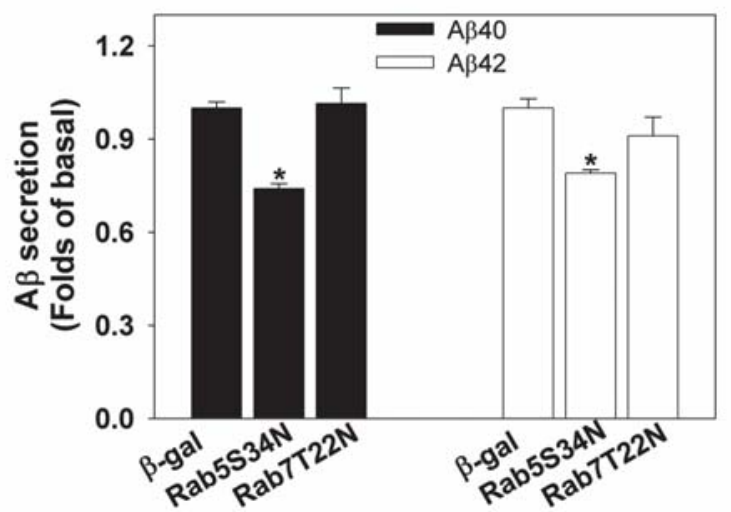

Figure 4. Role of endocytosis in APP-BACE1 age-dependent protein interactions. A. Average FRET efficiency (fold of the positive control) for coexpression of 3'myc-APP and 3'HA-BACE1 with B-gal, 5'flag-Rab5S34N or 5'flag-Rab7T22N in NHFs at PDLs 39, $\mathrm{n} \geq 15$. Data are from at least three independent experiments. ${ }^{*} \mathrm{P}<0.01$. B. NHFs at PDLs 39 were co-transfected 3'HA-BACE1 and 3'myc-APP with 3 -gal or 5'flag-Rab5S34N or 5'flagRab7T22N for $36 \mathrm{~h}$, then stained as follows: 3'HA-BACE1 (Cy3, red), 3'mycAPP (FITC, green) and EEA-1 for endosome (upper panel) or LAMP-1 for lysosome (lower panel) (Cy5, Blue). The immunofluorescence assay was used to detect the co-localization of 3'HA-BACE1 and 3'myc-APP with endosome or lysosome with 5'flag-Rab5S34N transfects (B3, B4), B-gal (B1, B2) and 5'flag-Rab7T22N (B5, B6) as the control of block efficiency. Scale bar, $40 \mu \mathrm{m}$. C. NHFs at PDLs 39 were co-transfected 3'HA-APP with B-gal or 5'flagRab5S34N or 5'flag-Rab7T22N for $48 \mathrm{~h}$, then accumulated cells with $500 \mu \mathrm{l}$ conditional media overnight, harvested the accumulated media for detection the secreted Aß40 and Aß42 level with ELISA. Each experiment was repeated at least three times. ${ }^{*} \mathrm{P}<0.01$. 
of amyloid plaque formation. Increases in APP, $\gamma$-secretase and BACE1 have been observed in cells and in vivo in correlation with age $(3,19)$. Other factors, such as level of serum cholesterol and phosphorylation of APP, which are associated with aging, may affect the function of $\beta$ - and $\gamma$-secretase (20). In this present study we show that production of $A B$ is increased in correlation with age in our cellular model of aging, as well as in vivo in rat hippocampi and human serum. Although opinions differ on the role of aging in $A \beta$ production, our data support that the increase of $A B$ production is age-related $(5,23)$. There is some mild discrepancy of age-related $\mathrm{A} \beta$ production between NHFs and our in vivo data. We attribute this discrepancy to differences in ratio of senescent cells translating from in vitro homogeneous cell-based systems to in vivo systems where cell populations are highly heterogeneous. Indeed, even at a very advanced age, animal and human brain tissues still contain significant populations of non-senescent cells (21).

There are several potential mechanisms for differential $A \beta$ production between healthy persons and those with AD (14), and the reason why increases occur with aging in both populations is the subject of intense investigation. Although APP-BACE1 protein-protein interaction as detected by FRET assay has been reported previously in cells driven to overexpress these proteins (7), we adopted NHFs cell-based aging model in order to study dynamic changes in this interaction. Our results show that APP-BACE1 interaction increases with cellular age, with the concomitant increase in $\mathrm{A} \beta$ production. One potential mechanism to explain this phenomenon is a dysregulation of APP metabolism occurring during the aging process, resulting in enhanced localization of APP in the endosome, where conditions are optimal for cleavage of APP by BACE1 $(3,7)$. Alternatively, increased APP-BACE1 interactions allow for more contact opportunities for cleavage reactions to occur (22), consequently causing a 'direct' increase in $A ß$ production (4). Moreover, given that there is elevated BACE1 activity in aged animal and human brains, this could also account for increased Aß levels (23). Data from this study indicate that APP-BACE1 interactions are necessary for the elevated $A \beta$ production, as a consequence of aging.

Given that endocytosis of both APP and BACE1 are correlated with $A ß$ production $(24,25)$, we hypothesized that APP-BACE1 interaction is a necessary first step for endocytosis of these two proteins. Using a dominant negative mutant of Rab5, we found that expression of this mutant significantly blocked APP-BACE1 interactions. Rab5 is a small G-protein known to be involved in the translocation of proteins from the cell membrane to the endosome (26). However, the dominant negative mutant of Rab7 that is known to contribute to lysosome formation (27) had no effect on APP-BACE1 association. These results suggest that the process of endosomal-targeted endocytosis is part of the specific mechanism of increasing APP-BACE1 protein interactions, as opposed to lysomal. Interestingly, both expression of endocytic factors and the rate of endocytosis are reported to increase with the process of aging $(28,29)$. While both endocytosis and autophagy potentially contribute to the aging process (30), our results indicate that APPBACE1 association is likely more involved as an endocytic process is needed.
With this study, we conclude that endocytosis is a significant molecular mechanism involved in the cellular aging process, an additional risk factor for AD pathology.

\section{Acknowledgements}

We thank A. Wiseman and P. Xia for revising the manuscript and S.M. Xin for technical assistance. This research was supported by grants from National Natural Science Foundation of China (30871103), China Post Doctoral Science Foundation and Shanghai Postdoctoral Science Foundation.

\section{References}

1. Tanzi RE: The synaptic Abeta hypothesis of Alzheimer disease. Nat Neurosci 8: 977-979, 2005.

2. Tanzi RE and Bertram L: Twenty years of the Alzheimer's disease amyloid hypothesis: a genetic perspective. Cell 120: 545-555, 2005.

3. Vassar R, Bennett BD, Babu-Khan S, Kahn S, Mendiaz EA, Denis P, Teplow DB, Ross S, Amarante P, Loeloff R, Luo Y, Fisher S, Fuller J, Edenson S, Lile J, Jarosinski MA, Biere AL, Curran E, Burgess T, Louis JC, Collins F, Treanor J, Rogers G and Citron M: Beta-secretase cleavage of Alzheimer's amyloid precursor protein by the transmembrane aspartic protease BACE. Science 286: 735-741, 1999.

4. Yan R, Bienkowski MJ, Shuck ME, Miao H, Tory MC, Pauley AM, Brashier JR, Stratman NC, Mathews WR, Buhl AE, Carter DB, Tomasselli AG, Parodi LA, Heinrikson RL and Gurney ME: Membrane-anchored aspartyl protease with Alzheimer's disease beta-secretase activity. Nature 402: 533-537, 1999.

5. Kern A, Roempp B, Prager K, Walter J and Behl C: Downregulation of endogenous amyloid precursor protein processing due to cellular aging. J Biol Chem 281: 2405-2413, 2006.

6. Mattson MP, Pedersen WA, Duan W, Culmsee C and Camandola S: Cellular and molecular mechanisms underlying perturbed energy metabolism and neuronal degeneration in Alzheimer's and Parkinson's diseases. Ann NY Acad Sci 893: 154-175, 1999.

7. Kinoshita A, Fukumoto H, Shah T, Whelan CM, Irizarry MC and Hyman BT: Demonstration by FRET of BACE interaction with the amyloid precursor protein at the cell surface and in early endosomes. J Cell Sci 116: 3339-3346, 2003.

8. Hayflick L and Moorhead PS: The serial cultivation of human diploid cell strains. Exp Cell Res 25: 585-621, 1961.

9. Cristofalo VJ: Cell culture aging: insights for cell aging in vivo? Aging 11: 1-3, 1999.

10. Campisi J, Dimri GP, Nehlin JO, Testori A and Yoshimoto K: Coming of age in culture. Exp Gerontol 31: 7-12, 1996.

11. Cristofalo VJ and Pignolo RJ: Molecular markers of senescence in fibroblast-like cultures. Exp Gerontol 31: 111-123, 1996.

12. Ni Y, Zhao X, Bao G, Zou L, Teng L, Wang Z, Song M, Xiong J, Bai Y and Pei G: Activation of beta2-adrenergic receptor stimulates gamma-secretase activity and accelerates amyloid plaque formation. Nat Med 12: 1390-1396, 2007.

13. Atamna H, Robinson C, Ingersoll R, Elliott H and Ames BN: $\mathrm{N}$-t-Butyl hydroxylamine is an antioxidant that reverses agerelated changes in mitochondria in vivo and in vitro. FASEB J 15: 2196-2204, 2001

14. Wang J, Dickson DW, Trojanowski JQ and Lee VM: The levels of soluble versus insoluble brain Abeta distinguish Alzheimer's disease from normal and pathologic aging. Exp Neurol 158: 328-337, 1999.

15. Huse JT, Pijak DS, Leslie GJ, Lee VM and Doms RW: Maturation and endosomal targeting of beta-site amyloid precursor protein-cleaving enzyme. The Alzheimer's disease beta-secretase. J Biol Chem 275: 33729-33737, 2000.

16. Gruenberg J: The endocytic pathway: a mosaic of domains. Nat Rev Mol Cell Biol 2: 721-730, 2001.

17. Daugherty BL and Green SA: Endosomal sorting of amyloid precursor protein-P-selectin chimeras influences secretase processing. Traffic 2: 908-916, 2001.

18. Hof PR: Morphology and neurochemical characteristics of the vulnerable neurons in brain aging and Alzheimer's disease. Eur Neurol 37: 71-81, 1997. 
19. Li Y, Zhou W, Tong Y, He G and Song W: Control of APP processing and Abeta generation level by BACE1 enzymatic activity and transcription. FASEB J 20: 285-292, 2006.

20. Pastorino L and Lu KP: Pathogenic mechanisms in Alzheimer's disease. Eur J Pharmacol 545: 29-38, 2006.

21. Sohrabji F and Bake S: Age-related changes in neuroprotection: is estrogen pro-inflammatory for the reproductive senescent brain? Endocrine 29: 191-197, 2006.

22. Repetto E, Russo C, Venezia V, Nizzari M, Nitsch RM and Schettini G: BACE1 overexpression regulates amyloid precursor protein cleavage and interaction with the ShcA adapter. Ann NY Acad Sci 1030: 330-338, 2004.

23. Fukumoto H, Rosene DL, Moss MB, Raju S, Hyman BT and Irizarry MC: Beta-secretase activity increases with aging in human, monkey, and mouse brain. Am J Pathol 164: 719-725, 2004.

24. Selkoe DJ, Yamazaki T, Citron M, Podlisny MB, Koo EH Teplow DB and Haass C: The role of APP processing and trafficking pathways in the formation of amyloid beta-protein Ann NY Acad Sci 777: 57-64, 1996.

25. Huang XP, Chang WP, Koelsch G, Turner RT 3rd, Lupu F and Tang J: Internalization of exogenously added memapsin 2 (betasecretase) ectodomain by cells is mediated by amyloid precursor protein. J Biol Chem 279: 37886-37894, 2004.
26. Sterling H, Lin DH, Gu RM, Dong K, Hebert SC and Wang WH: Inhibition of protein-tyrosine phosphatase stimulates the dynamin-dependent endocytosis of ROMK1. J Biol Chem 277: 4317-4323, 2002.

27. Ahn S, Kim J, Lucaveche CL, Reedy MC, Luttrell LM, Lefkowitz RJ and Daaka Y: Src-dependent tyrosine phosphorylation regulates dynamin self-assembly and ligand-induced endocytosis of the epidermal growth factor receptor. J Biol Chem 277: 26642-26651, 2002.

28. Levine B and Klionsky DJ: Development by self-digestion: molecular mechanisms and biological functions of autophagy. Dev Cell 6: 463-477, 2004.

29. Cataldo AM, Peterhoff CM, Troncoso JC, Gomez-Isla T, Hyman BT and Nixon RA: Endocytic pathway abnormalities precede amyloid beta deposition in sporadic Alzheimer's disease and Down syndrome: differential effects of APOE genotype and presenilin mutations. Am J Pathol 157: 277-286, 2000.

30. Donati A: The involvement of macroautophagy in aging and anti-aging interventions. Mol Aspects Med 27: 455-470, 2006. 